\title{
The Performance Assessment of a Semisubmersible Platform Subjected to Wind and Waves by a CFD/6-DOF Approach
}

\author{
Hongbo Zhu, ${ }^{1}$ Dai Zhou $\mathbb{D}^{1},{ }^{1,2,3}$ Zhaolong Han $\mathbb{D}^{1,},{ }^{1,3}$ Jin $\mathrm{Ma}^{4}{ }^{4}$ Yan Bao, ${ }^{1,3}$ and Shixiao Fu ${ }^{1,2,5}$ \\ ${ }^{1}$ School of Naval Architecture, Ocean and Civil Engineering, Shanghai Jiao Tong University, Shanghai 200240, China \\ ${ }^{2}$ State Key Laboratory of Ocean Engineering, Shanghai 200240, China \\ ${ }^{3}$ Shanghai Key Laboratory for Digital Maintenance of Buildings and Infrastructure, Shanghai 200240, China \\ ${ }^{4}$ Shanghai Construction Group (SCG), Shanghai 200120, China \\ ${ }^{5}$ Key Laboratory of Hydrodynamics of Ministry of Education, Shanghai 200240, China
}

Correspondence should be addressed to Dai Zhou; zhoudai@sjtu.edu.cn and Zhaolong Han; han.arkey@sjtu.edu.cn

Received 4 June 2020; Revised 29 June 2020; Accepted 27 July 2020; Published 19 August 2020

Academic Editor: Hong-jun Zhu

Copyright (C) 2020 Hongbo Zhu et al. This is an open access article distributed under the Creative Commons Attribution License, which permits unrestricted use, distribution, and reproduction in any medium, provided the original work is properly cited.

\begin{abstract}
The semisubmersible platform is one of the most important equipment for the exploitation of deep-sea oil and gas. And its survival and operational capacities depend on the towing and motion performances. The present work investigates the properties of different platform component designs based on their towing and motion performances under wind and waves by using an integrated computational fluid dynamics (CFD) method. The joint application of RANS and a VOF/6-DOF solver compiled in a new Fluent UDF Library is adopted for the main calculation. Numerical simulations and results demonstrate that the VOF/6-DOF solver is accurate and stable, which could achieve the same functions as the previous OpenFOAM scheme. In order to acquire a better towing performance, different shapes are suggested for the pontoon end shape, the column cross section, and the brace longitudinal section, respectively. As to the motion response, among all the three design selections, the column cross section has the greatest influence on the whole platform. The simulation results reveal that circle section increases the roll and pitch response and reduces the heave response, to which the square section is adverse.
\end{abstract}

\section{Introduction}

Because of their limited deployment range, fixed platforms could hardly meet the need of deep exploitation of marine source, which includes renewable energy, oil and gas, et al.; recent focus has therefore been shifted to the floating offshore platforms, especially the semisubmersible offshore platform [1]. At present, it can serve as a drilling production platform or an offshore wind turbine foundation [2]. The towing and motion performances, which are among the vital indicators of the offshore platform performance, will largely affect the operation efficiency and safety of a semisubmersible platform [3]. Hence, it is important to investigate the effects of different platform component designs on the towing and motion performances.

The effects of wind, waves, and currents on the platform performance have attracted a number of experimental, field, and numerical investigations [4-7]. In general, experiments and field tests may have some limitations. For instance, they are usually too expensive to be performed for one type of semisubmersible platform. Further, the field test also requires the harsh environmental conditions and test devices. For a full-scale platform or a scaled platform model, not all tanks are sufficiently large to perform model test of a floating platform. Regarding the numerical investigation aspect, with the enhancement of computing capability, a number of numerical methods and software packages (e.g., AQWA [8], Fluent [9], WAMIT [10], and FAST [11]) are currently available for time- and frequency-domain analyses of a floating platform under wind and waves, etc. However, in several software packages (e.g., ANSYS/Fluent), the dynamic characteristics and relations between wind and waves are still not well considered [12]. In order to improve this problem, we developed a computational program 
incorporating the wind loading and the stochastic waves based on our previous studies [13] and [14]. It was realized by integrating modules of MATLAB, ANSYS, and Fluent to investigate the motion and towing performances.

Computational fluid dynamics (CFD) combined with the volume of fluid (VOF)/6-DOF solver is a powerful tool for investigating rigid-body motion hydrodynamics. According to a comparison study, the unsteady CFD simulations could capture the complete physical phenomena (motion response, towing resistance, wave run-up, etc.) of a floating platform [2]. In particular, just by reading the geometry and mass properties of the platform into the computational code, a CFD simulation was effective to provide the accurate solutions [15]. Further, a number of 6-DOF solvers have been developed over the past 20 years [16] and applied to record aircraft, ship, and submarine motions [17-20]. And more recently, it expands to offshore platform dynamics [21-23]. However, the computational instabilities caused by the artificial added mass were hard to be resolved in these traditional 6-DOF solvers $[24,25]$. In some open-source CFD software packages, such as OpenFOAM, a 6-DOF solver is able to be implemented to form a tightly coupled algorithm for simulating wave slamming on ship hulls and a wind turbine platform $[15,26,27]$. The objective of the present study is to assess the effects of different structural component designs on the towing and motion performances under the wind and waves. By compiling a VOF/6-DOF solver in a Fluent UDF Library, a tightly coupled CFD/6-DOF algorithm is constructed and used to monitor the towing resistance and motion response of a semisubmersible platform under the combined wind and wave loadings.

The paper is organized as follows: the structural models together with their corresponding parameters are presented in Section 2; numerical methods for conducting CFD simulations are provided in Section 3; validations for mesh strategy and a VOF/6-DOF solver are included in Section 4; and numerical evaluations of towing and motion performances are conducted in Section 5.

\section{A Semisubmersible Platform}

2.1. Platform Model. The original platform model is a 6thgeneration semisubmersible platform, primarily composed of a deck, four columns, two pontoons, and four braces. As is focusing on the towing and motion performances assessment, the derrick and other deck structures are simplified as mass sources acting on the deck. The platform model is established in ANSYS/Workbench as shown in Figure 1. The dimensions and survival conditions of this model are listed in Tables 1 and 2. It is noted that the center of gravity of the simplified model will be changed in Section 3.2 by ANSYS/ AQWA software.

2.2. The Definition of the Component Form. The in-depth investigations are focused on the effects of different structural patterns on the platform's towing and motion performances. Numerical simulations are performed on three types of existing semisubmersible platform components: the

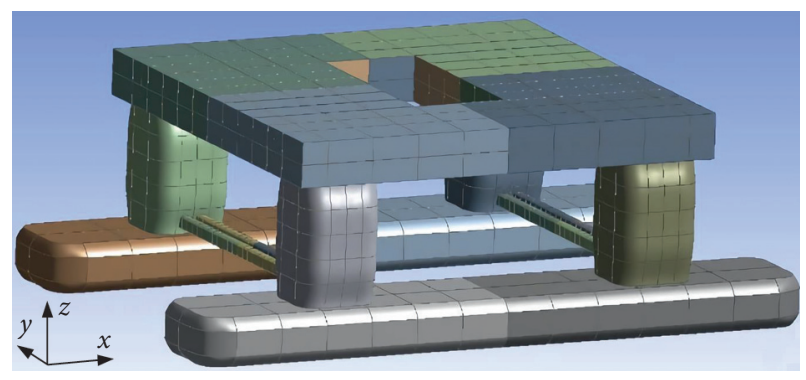

Figure 1: The basic model of a semisubmersible platform.

Table 1: The dimensions of the semisubmersible platform model.

\begin{tabular}{lc}
\hline Members & Dimensions $($ length $\times$ width $\times$ height $)(\mathrm{m})$ \\
\hline Pontoon & $114.07 \times 20.12 \times 8.54$ \\
Column & $17.39 \times 17.39 \times 21.46$ \\
Deck & $74.42 \times 74.42 \times 8.6$ \\
Brace & $42.7 \times 1.83$ \\
\hline
\end{tabular}

TABLE 2: The parameters in survival conditions.

\begin{tabular}{lc}
\hline Draft $(\mathrm{m})$ & 16.0 \\
Air gap (m) & 14.0 \\
The height of the center of gravity from the surface $(\mathrm{m})$ & 8.8 \\
Displacement (ton) & 48206.8 \\
\hline
\end{tabular}

pontoon end shapes, the cross section of the columns, and the longitudinal section of the braces. Based on the original platform model in Figure 1, six numerical cases and their corresponding parameters are presented in the following.

Table 3 lists the structural parameters of three common pontoon end shapes in the same displacement: half round, sharp angle, and rounded cube, which are in turn defined as Case 1, Case 2, and Case 3. The corresponding structural forms are shown in Figure 2. Case 3 is the basic platform model as in Figure 1, where the sharp angle is $30^{\circ}$ and chamfer radius is $6 \mathrm{~m}$.

Based on the model of Case 3, the sections of the columns for Case 3 and Case 4 are modified as shown in Table 4 , which are square and circle cross sections, respectively. Moreover, the pontoon end shapes and the longitudinal section of the braces are, respectively, rounded cube and circle.

Finally, three longitudinal sections of the braces are recorded in Table 5, which are the circle (Case 3), the halfround plate shape (Case 5), and the plate shape with the chamfer radius of $0.3 \mathrm{~m}$ (Case 6). In the subsimulations, the pontoon end shapes and the cross section of the columns are rounded cube for Case 5 and circle for Case 6 . In view that these sections are not complicated, the platform models of different column and brace sections are not displayed here.

\section{Computational Method}

3.1. Computational Fluid Dynamics (CFD). CFD simulations are conducted in the platform of ANSYS/Fluent 17.0, coupled with the User Defined Functions (UDFs) 
TABLE 3: The structural parameters of three pontoon end shapes.

\begin{tabular}{lccc}
\hline & Case 1 & Case 2 & Case 3 \\
\hline End shape & Half round & $30^{\circ}$ sharp angle & Rounded cube \\
The length of the pontoon $(\mathrm{m})$ & 116.85 & 130.23 & 114.07 \\
\hline
\end{tabular}

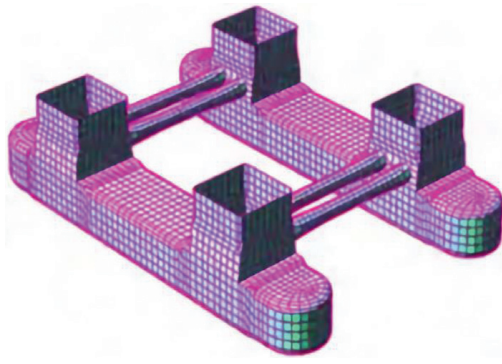

(a)

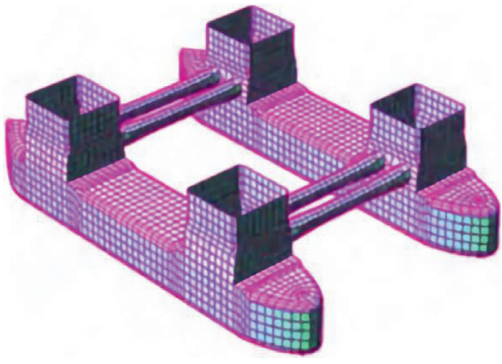

(b)

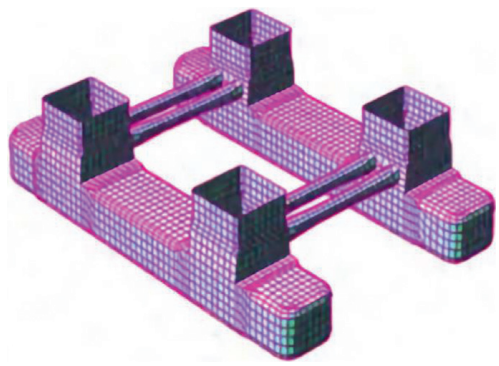

(c)

Figure 2: The platform models with three pontoon end shapes. (a) Case 1: half-round model; (b) Case 2: sharp-angle model; and (c) Case 3: rounded-cube model.

TABLE 4: The structural parameters of two column sections.

\begin{tabular}{lcr}
\hline & Case 3 & Case 4 \\
\hline Cross section & Square & Circle \\
Dimension $(\mathrm{m})$ & 17.39 (side length) & 9.81 (radius) \\
\hline
\end{tabular}

TABle 5: The structural parameters of three brace sections.

\begin{tabular}{lccc}
\hline & Case 3 & Case 5 & Case 6 \\
\hline Longitudinal section & Circle & Half-round plate shape & Plate shape with the chamfer radius of $0.3 \mathrm{~m}$ \\
Circumference $(\mathrm{m})$ & 11.49 & 16.78 & 14.64 \\
\hline
\end{tabular}

techniques. These numerical simulations are completed by solving the unsteady Reynolds-averaged Navier-Stokes (RANS) equations. The discrete equations are solved by the pressure-implicit with splitting of operators (PISO) method. The time and space discretizations are both treated by second-order discrete schemes. Bounded central differencing is adopted for momentum discretization. Herein, the shear stress transport (SST) $k$ - $\omega$ model, which is applicable in many CFD simulations [12], is utilized to resolve the turbulent behaviors. The governing equations of the SST $k-\omega$ turbulence model are listed as below:

$$
\left\{\begin{array}{l}
\rho\left(\frac{\partial u_{i}}{\partial t}+\frac{\partial u_{i} u_{j}}{\partial x_{j}}\right)=-\frac{\partial p}{\partial x_{i}}+\frac{\partial}{\partial x_{j}}\left(\Gamma \frac{\partial u_{i}}{\partial x_{j}}\right)+S_{i} \\
\rho\left(\frac{\partial k}{\partial t}+\frac{\partial k u_{j}}{\partial x_{j}}\right)=\frac{\partial}{\partial x_{j}}\left(\Gamma_{k} \frac{\partial k}{\partial x_{j}}\right)+G_{k}-Y_{k}+S_{k} \\
\rho\left(\frac{\partial \omega}{\partial t}+\frac{\partial \omega u_{j}}{\partial x_{j}}\right)=\frac{\partial}{\partial x_{j}}\left(\Gamma_{\omega} \frac{\partial k}{\partial x_{j}}\right)+G_{\omega}-Y_{\omega}+D_{\omega}+S_{\omega}
\end{array}\right.
$$

where $\rho$ is the fluid density, $p$ is the fluid pressure, $u$ is the fluid velocity, $k$ is the turbulent kinetic energy, $\omega$ is the dissipative rate, $\Gamma, \Gamma_{k}$, and $\Gamma_{\omega}$ are, respectively, the effective diffusion coefficients of $u, k$, and $\omega, S_{i}, S_{k}$, and $S_{\omega}$ are the custom source items of the three abovementioned equations, $Y_{k}$ and $Y_{\omega}$ are the dissipative terms of $k$ and $\omega$, and $D_{\omega}$ is the cross-diffusion term.

3.2. A VOF/6-DOF Solver. To describe the motion and towing performances of a semisubmersible offshore platform under the combined wind and wave loadings, the volume of fluid (VOF) model together with a tightly coupled CFD/6-DOF solver, which is named as the VOF/6-DOF solver, is employed. A self-compiled Fluent User-Defined Function Library is developed to effectively realize the transient interface motion.

VOF model is suitable for the analyses of two immiscible fluid phases (i.e., wind and waves). Under the assumption that wind and waves can share velocity and pressure fields, the governing equations describing the momentum, mass, and energy transport are solved for an equivalent fluid, which regards the whole domain as a single-phase fluid. Therefore, the transport equation of the volume fluid is treated as follows: 


$$
\frac{\partial V_{i}}{\partial t}+\frac{\partial V_{i} u_{x}}{\partial x}+u_{y} \frac{\partial V_{i} u_{y}}{\partial y}+u_{z} \frac{\partial V_{i} u_{z}}{\partial z}=\frac{\mathrm{MST}}{\rho_{i}}
$$

where $V_{i}$ is the volume fraction for the fluid $i(i=1,2)$. The total volume fraction in one controlled volume is unit (i.e., $\left.V_{1}+V_{2}=1\right) \cdot u_{x}, u_{y}$, and $u_{z}$ are the velocity components of $\boldsymbol{u}$ in the Cartesian coordinates $\mathrm{O}_{x y z}$, whose origin is at the interface. MST is the additional mass source, and $\rho_{i}$ is the density of the corresponding fluid $i$.

Later on, in the CFD simulations, the platform is considered as a 6-DOF rigid body, whose motion could be decomposed into the translation and rotation as depicted in Figure 3(a). Thus, the transient position of the platform body refers to that of the center of mass $\boldsymbol{x}_{r}$, whose rotation motion is sketched in Figure 3(b). Its instantaneous motion position update is recorded by the following equation:

$$
\left\{\begin{array}{l}
\mathbf{x}_{r}^{i+1}=\mathbf{x}_{r, r}^{i+1}+\mathbf{x}_{r, t}^{i+1}, \\
\mathbf{x}_{r, t}^{i+1}=\mathbf{x}_{p}^{i}+\mathbf{v}_{p} \Delta t \\
\mathbf{x}_{r, r}^{i+1}=\mathbf{x}_{r, r}^{i}+\left|\mathbf{x}_{r, r}^{i}\right|\left\{\sin (\Delta \theta) \mathbf{e}_{t}+[\cos (\Delta \theta)-1] \mathbf{e}_{n}\right\}
\end{array}\right.
$$

where $\boldsymbol{x}_{r, t}$ and $\boldsymbol{x}_{r, r}$ are, respectively, the motion position of the translation and rotation, $x_{p}$ is the position of the center of mass, $\mathbf{v}_{p}$ is the linear velocity, $\boldsymbol{\omega}_{p}$ is the angular velocity, $\Delta \theta$ is the rotation angle from time $i$ to time $i+1$, and $\boldsymbol{e}_{t}$ and $\boldsymbol{e}_{n}$ are, respectively, tangential and normal unit vectors.

Different from a traditional 6-DOF solver, a relaxation factor $\eta$ is applied to relax body force $F$ and moment $M$, which is described as follows [15]:

$$
\left\{\begin{array}{l}
\widetilde{F}=F_{i-1}+\eta_{i}\left(F_{i}-F_{i-1}\right), \\
\widetilde{M}=M_{i-1}+\eta_{i}\left(M_{i}-M_{i-1}\right),
\end{array}\right.
$$

where the sign " $\sim$ " refers to the corresponding relaxed value. Combined with the Aitken method conducting dynamic relaxation, the motion response/towing resistance at each time step is recorded before the next time step, which can effectively avoid the calculation instabilities in the traditional 6-DOF solving algorithms.

The algorithm of the present VOF/6-DOF solver in the iterative calculations is concluded as follows:

Step 1. Solve body forces in the equivalent fluid field.

Step 2. Relax body forces and moments by (4).

Step 3. Solve 6-DOF motion equation (3).

Step 4. Update the mesh based on the new transient position.

Step 5. Correct fluid field variables for mesh motion.

Step 6. Solve VOF equation (2).

Step 7. Record the towing resistance or motion response by UDFs.
Step 8. Check the solution convergence and update the initial relaxation factor and return to 1 .

\subsection{Computational Parameters}

3.3.1. Fluid domain. Before establishing the computational domain, the center of gravity of this simplified platform is determined by using ANSYS/AQWA. Taking Case 3 as an example, the position changes of the center of gravity in still water within 4 iterations are depicted in Figure 4. As the center of gravity is raised by $0.22 \mathrm{~m}$, the air gap is modified as $14.22 \mathrm{~m}$ accordingly. By using this method, the relationship between the relative positions of the interface and each platform in Cases 1 to 6 can be calculated.

The CFD simulations of the full-scale semisubmersible platform models are performed in a hexahedral computational domain with a dimension of $1200 \mathrm{~m} \times 500 \mathrm{~m} \times 300 \mathrm{~m}$ (length $\times$ width $\times$ height) shown in Figure 5(a). The platform model is located $300 \mathrm{~m}$ away from the inlet. And tetrahedral grids are generated in the neighborhood of the model surfaces as in Figure 5(b). The maximum and minimum grid lengths are, respectively, $0.65 \mathrm{~m}$ and $0.11 \mathrm{~m}$, and the grid stretching ratio is kept to be less than 1.05. In consideration of the computer capability and time cost, the minimum values of $y+$ is less than 3.5 , which could be enough to satisfy engineering requirements [28]. The total grid numbers from Cases 1 to 6 are presented in Table 6 . All the computation procedures are carried out on a computer server with $\mathrm{CPU}$ type of Intel Xeon E3-1220 v3 and $3.1 \mathrm{GHz}$ with a $32 \mathrm{G}$ memory.

The UDF Library of the VOF/6-DOF solver requires the structural mass, the moment of inertia, and other parameters, which are also computed in ANSYS/AQWA. According to Geng's [29] previous work, the following equation can be utilized for the total mass, the centroid coordinates, and the moment of inertia, which is written as

$$
[M]=\left[\begin{array}{cccccc}
M & 0 & 0 & 0 & M\left(z_{c}-z_{0}\right) & M\left(y_{c}-y_{0}\right) \\
0 & M & 0 & -M\left(z_{c}-z_{0}\right) & 0 & M\left(x_{c}-x_{0}\right) \\
0 & 0 & M & -M\left(y_{c}-y_{0}\right) & -M\left(x_{c}-x_{0}\right) & 0 \\
& & I_{22}+I_{33} & -I_{12} & -I_{13} \\
& 0 & -I_{12} & I_{33}+I_{11} & -I_{23} \\
& & & -I_{13} & -I_{23} & I_{22}+I_{11}
\end{array}\right],
$$

where $M$ is the total mass, $I_{\mathrm{ij}}$ is the moment of inertia, $x_{c}, y_{c}$, and $z_{c}$ are the centroid coordinates in the present position, and $x_{0}, y_{0}$, and $z_{0}$ is the centroid coordinates in the initial position. For instance, the mass matrix [M] of Case 3 is obtained as follows: 


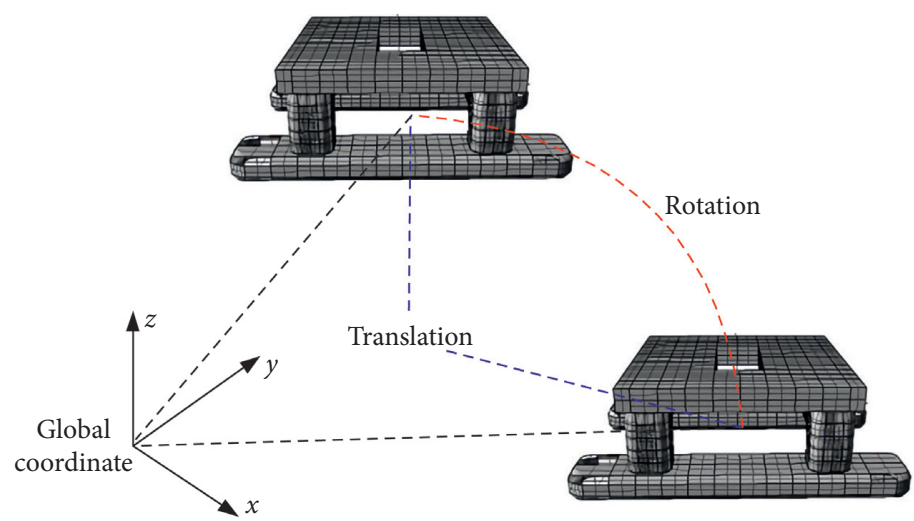

(a)

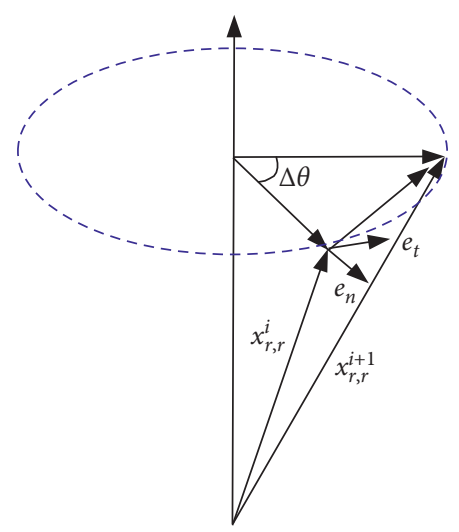

(b)

Figure 3: (a) The 6-DOF motion of the platform. (b) The rotation motion of the center of mass.

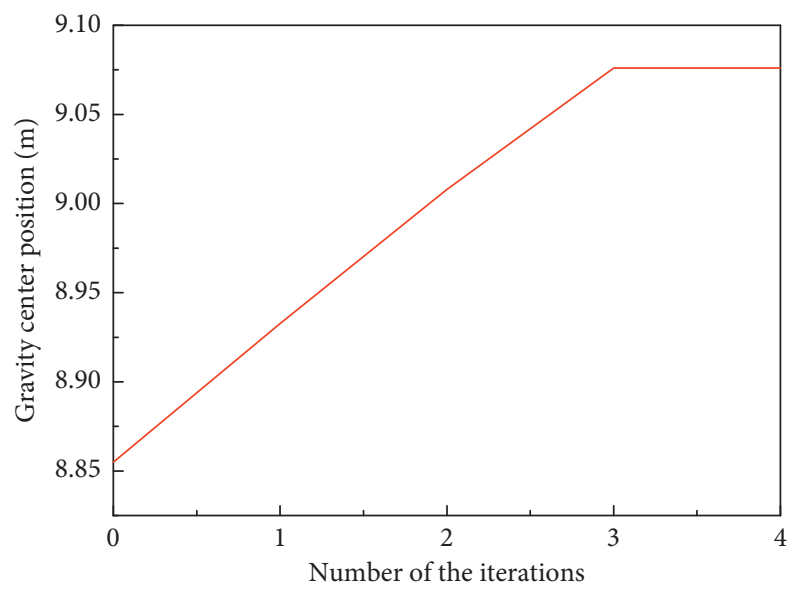

Figure 4: The iterative results of the center of gravity of a semisubmersible platform (Case 3) in still water.

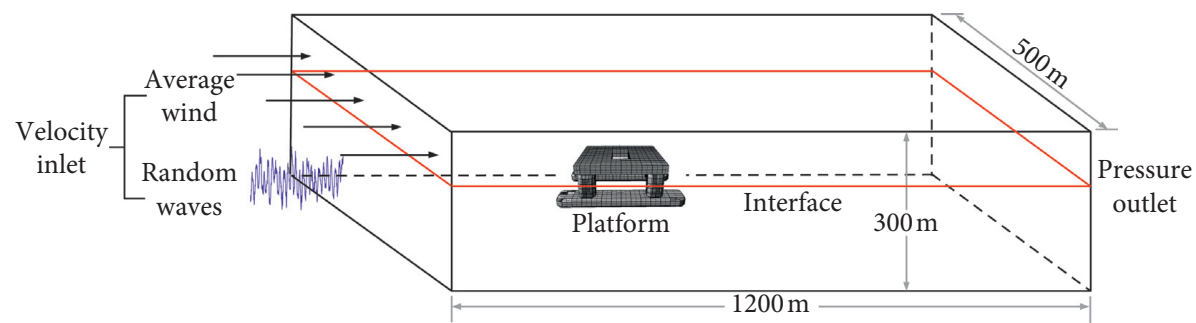

(a)



(b)

Figure 5: (a) A sketch of the 3D computational fluid domain. (b) Full grid domain.

Table 6: The total grid number of six numerical cases.

\begin{tabular}{lcccccc}
\hline & Case 1 & Case 2 & Case 3 & Case 4 & Case 5 & Case 6 \\
\hline Grid no. & 4637636 & 5027036 & 4015685 & 4028057 & 4007036 & 4019690 \\
\hline
\end{tabular}




$\left[\begin{array}{cccccc}5.9154 \times 10^{6} & 0 & 0 & 0 & 9.852 \times 10^{7} & 29.548 \\ 0 & 5.9154 \times 10^{6} & 0 & -9.852 \times 10^{7} & 0 & 100.94 \\ 0 & 0 & 5.9154 \times 10^{6} & -29.548 & -100.94 & 0 \\ & & & 2.7424 \times 10^{9} & -9189.4 & -1166.5 \\ & 0 & & -9189.4 & 2.833 \times 10^{9} & 391.15 \\ & & & -1166.5 & 391.15 & 1.3135 \times 10^{9}\end{array}\right]$

The parameters of other cases in the VOF/6-DOF solver are calculated in the same way.

3.3.2. The Boundary Conditions. As shown in Figure 5, the velocity inlet and pressure outlet are included in the computational domain. Thus, the present study could make use of the velocity inlet to read the time series of wave speed and the average wind speed by the self-compiled UDFs and VOF model, which is sketched in Figure 5(a).

On the basis of our previous study [14], the relationship between the wind and waves (i.e., the relationship of the average wind speed $V_{0}$ and the significant wave height $H_{s}$ ) is explored to guarantee that the strength of wind and waves could match. The relations between $H_{s}$ and $V_{0}$ and the derived power spectral density function of waves $S(\omega)$ are, respectively, written as

$$
\begin{aligned}
H_{s}= & 0.27355-0.21802 V_{0}+0.05538 V_{0}^{2}-0.00121 V_{0}^{3} \\
& +0.0000155239 V_{0}^{4}, \\
S(\omega)= & \sqrt{\frac{\rho_{1}}{2 \rho_{2} g^{2}}} \cdot V_{0} \cdot \Phi^{3 / 2} \cdot \beta_{1}(\Phi)^{1 / 2} \cdot A(\Phi) \cdot \gamma,
\end{aligned}
$$

where $\Phi=\vartheta \omega / \omega_{p}$ is the modified natural frequency, $\vartheta$ is a corrected coefficient of $\omega, \omega_{p}$ is the peak frequency, $A(\omega)$ is the amplitude, $\gamma$ is a corrected coefficient of $A(\omega)$, and $\beta(\omega)$ is the energy transferring coefficient. The time series of wave velocity is generated by the MS method and self-compiled MATLAB programs, which have been validated and applied in our previous studies.

The computational procedure of the velocity inlet is provided as follows.

Step 1. Determine $\mathrm{V}_{0}$ and the corresponding wave PSD by (7) and (8)

Step 2. Generate the time series of wave speed of the nodes at the inlet

Step 3. Read $V_{0}$ by the VOF model and the time series of waves by the self-compiled UDFs at the $i$ th time step

Step 4. Conduct the CFD calculation at the ith time step

\section{Validation Tests}

In this section, three types of validation tests are performed: the grid independence tests, the time step independence tests, and the VOF/6-DOF solver test. Firstly, the grid and time step independence tests are conducted to balance the time cost with the computing accuracy. Then, the VOF/6DOF solver compiled in a Fluent UDF Library is verified to be applicable in the present numerical simulations. It should be noted that the object of all these validations is based on Case 3 whose sea state, including average wind speed, significant wave height, and wave period, is given as $11 \mathrm{~m} / \mathrm{s}$, $3 \mathrm{~m}$, and $6 \mathrm{~s}$, respectively.

4.1. The Grid Independence Tests. To verify the grid generation method and its implementation in the present study, we refine the computational mesh and perform the CFD simulations by using four types of mesh: the coarse mesh, mesh given in the present study, the medium mesh, and the fine mesh, which contain approximately $3 \times 10^{6}, 4 \times 10^{6}$, $5 \times 10^{6}$, and $6 \times 10^{6}$ computational cells, respectively.

Figures 6 and 7 show the towing and motion performances assessment by using these four types of mesh. As seen from Figures 6(a) and 7(a), all the curves of four types of mesh have a relatively similar trend, which preliminarily illustrates the reliability of the computational results by using the present grid generation method. Furthermore, Figures 6(b) and 7(b) investigate the effect of the mesh density based on the average error and time cost. In Figure 6(b), compared to the present study, the average error of the coarse mesh is larger than that of the medium and fine mesh, which are $11.8 \%, 4.27 \%$, and $4.18 \%$, respectively. It is also found that the numerical accuracy has no significant improvement with the increasing grid number. However, with regard to the time cost ratio, shown as the top $x$-axis and the right $y$-axis in Figure 6(b), time cost is increasing rapidly with the grid number.

In addition, to further quantify the influence of different meshes on heave response, the Root Mean Square (RMS) of heave response $\left(Z_{R M S}\right)$ of four mesh methods is analyzed in the present section. As given in Table 7, with the increase of the number of meshes, the results show a tendency of convergence. A discrepancy of only $0.29 \%$ is noticed when mesh type changes from medium to fine method. Combined with the above analysis, it could be approved that the grid generation method in the present study is reasonable and applicable in the following numerical simulations.

4.2. The Time Step Independence Tests. To demonstrate the time step setting, we also perform the CFD simulations by using three different time steps: $0.01 \mathrm{~s}$ (the present study), $0.005 \mathrm{~s}$, and $0.0025 \mathrm{~s}$. Similarly, Figures 8 and 9 show the 


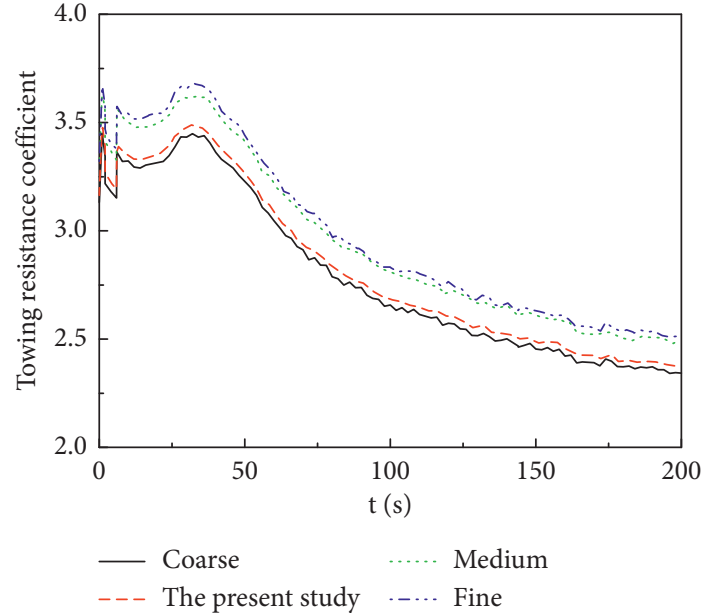

(a)

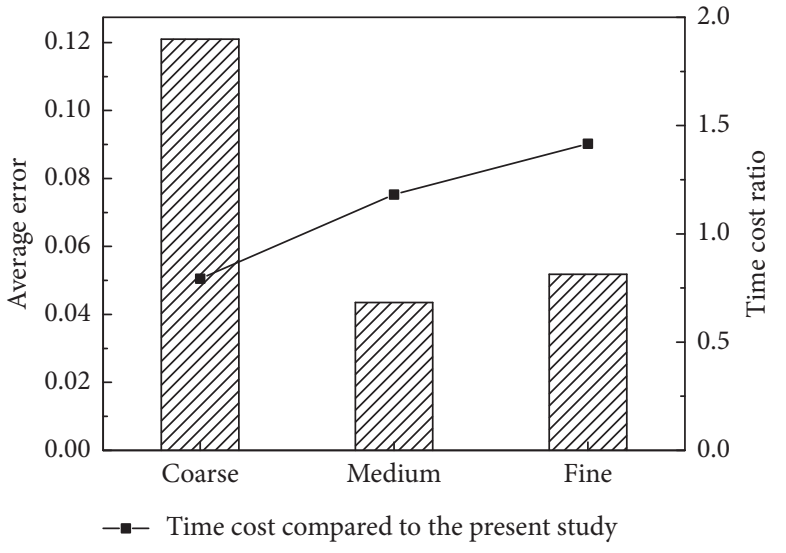

(b)

FIGURE 6: The towing performance comparisons of different mesh methods: (a) the time series of towing resistance coefficient at the towing speed of $4 \mathrm{kN}$ (represented by the speed of the fluid) and (b) the average error and time cost ratio statistics compared to the present study.

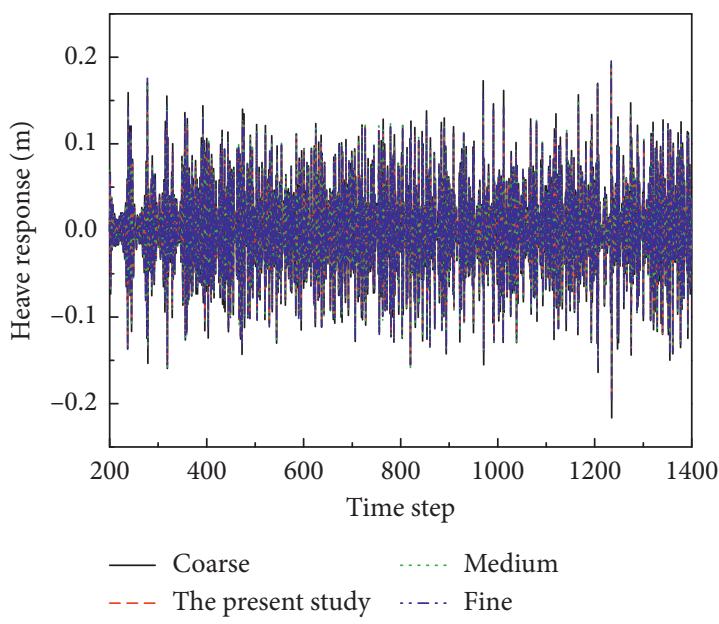

(a)

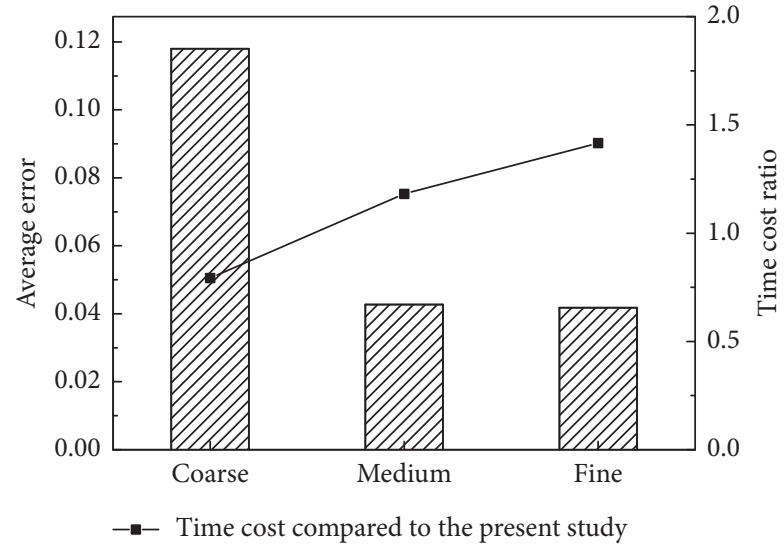

(b)

FIGURE 7: The heave response comparisons of different mesh methods: (a) the time series of heave displacement and (b) the average error and time cost ratio statistics compared to the present study.

TABLE 7: RMS of heave response of different mesh methods.

\begin{tabular}{ccccc}
\hline Case & Coarse & Present study & Medium & Fine \\
\hline \multirow{2}{*}{$Z_{\text {RMS }}$} & $\begin{array}{c}0.0689 \\
(0.58 \%)\end{array}$ & 0.0685 & $0.0684(0.15 \%)$ & $0.0683(0.29 \%)$ \\
\hline
\end{tabular}

evaluation of the towing and motion performances of these three types of time step.

As depicted in Figures 8(a) and 9(a), all the curves have a relatively similar trend, which depicts that $\Delta t=0.01 \mathrm{~s}$ is an applicable time step. Then, the average error and time cost ratio statistics in Figures 8(b) and 9(b) show that the numerical accuracy has little change with the decreasing time step (i.e., both two average errors are less than 5\%).
However, the time cost has a rapid increase with the time step. Besides, Table 8 further states that numerical accuracy will not be sensitive to a smaller time step. Therefore, in a comprehensive consideration of the numerical accuracy and efficiency, the present time step is applicable in the following numerical simulations.

4.3. The VOF/6-DOF Solver. Dunbar et al. [15] developed and validated a tightly coupled CFD/6-DOF method with better accuracy and stability by OpenFOAM through the motion performance investigations of a floating offshore wind turbine platform by comparing three methods. In consideration of the larger size and more complex structures of the present production platform, we perform two types of numerical simulations to validate the accuracy and stability 


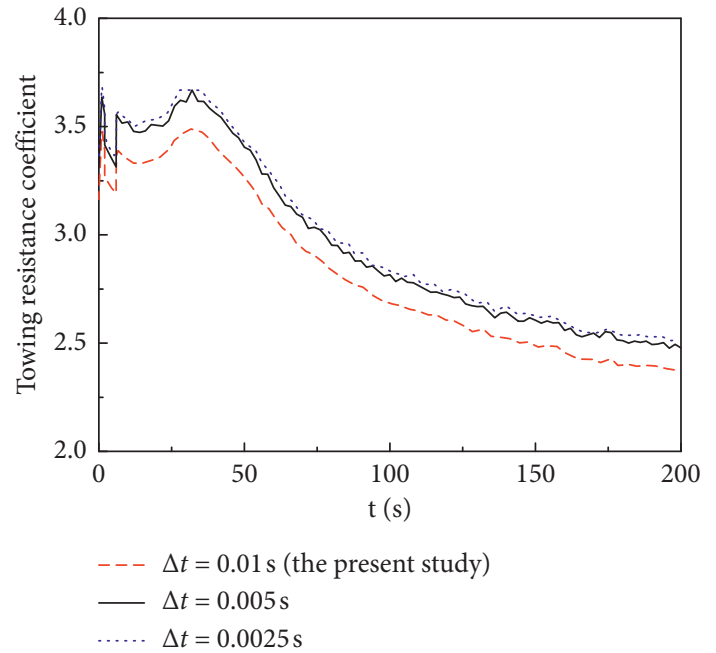

(a)

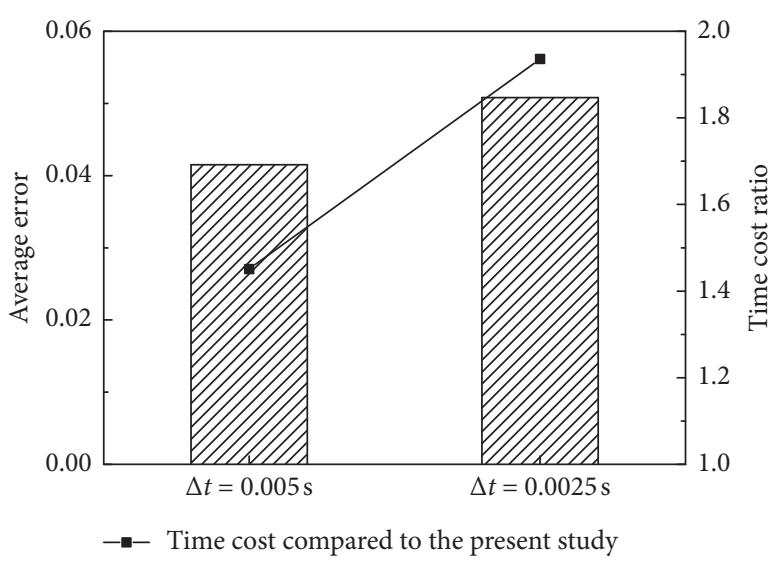

(b)

FIGURE 8: The towing performance comparisons of different time steps: (a) the time series of towing resistance coefficient at the towing speed of $4 \mathrm{kN}$ and (b) the average error and time cost ratio statistics compared to the present study.

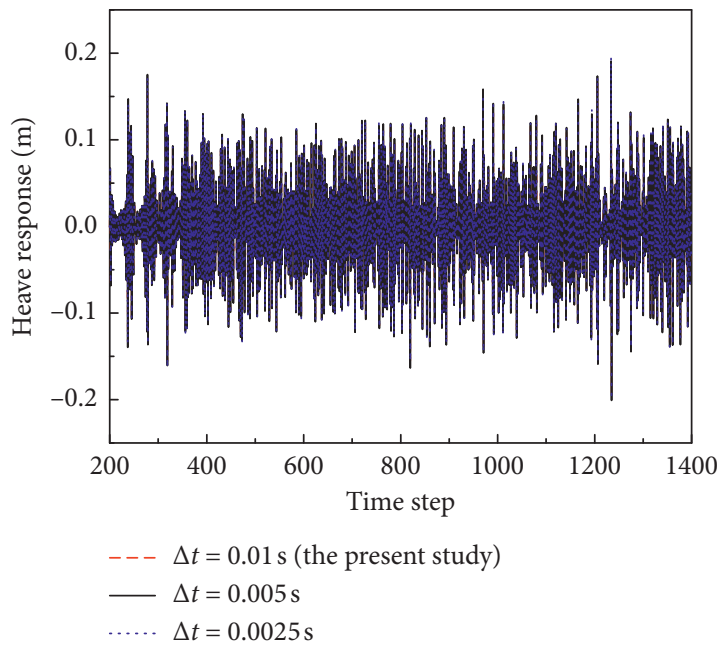

(a)

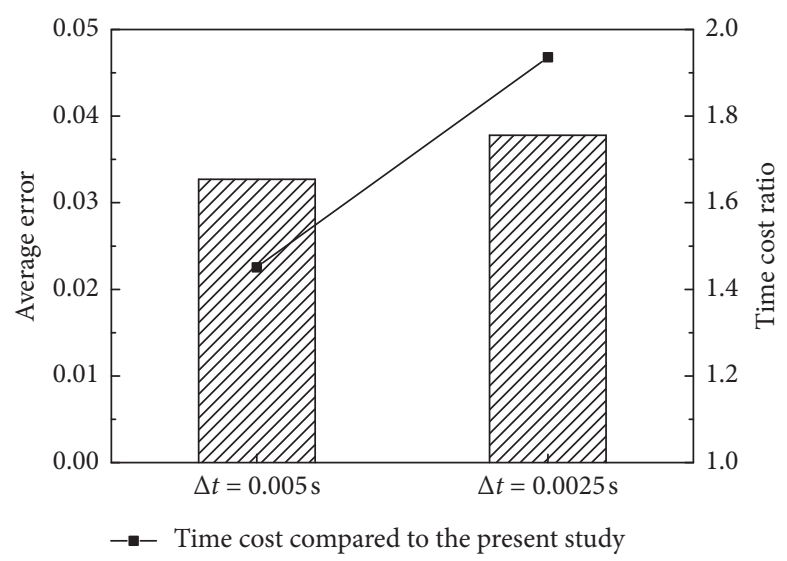

(b)

FIGURE 9: The heave response comparisons of different time steps: (a) the time series of heave displacement and (b) the average error and time cost ratio statistics compared to the present study.

TABLE 8: RMS of heave response of different time steps.

\begin{tabular}{lccc}
\hline Case & $\Delta t=0.01$ (present study) & $\Delta t=0.005$ & $\Delta t=0.0025$ \\
\hline$Z_{R M S}$ & 0.0685 & 0.0685 & $0.0684(0.15 \%)$ \\
\hline
\end{tabular}

of the VOF/6-DOF solver compiled in the present Fluent UDF Library.

4.3.1. Numerical Simulation I. The offshore wind turbine platform is selected for validation tests. Figure 10(a) plots the time series of heave displacement by ANSYS/Fluent adopted in the present method and OpenFOAM. It is obviously found that the present results are relatively in agreement with the previous studies $[12,15]$. Both curves have the relatively stable and similar oscillations, and only several peak and valley values are a little different. The in-depth error analyses in Figure 10(b) show that the average error is approximately $7 \%$, which is acceptable to be within the engineering criteria. Thus, the accuracy and stability of the present method and implementation could be validated.

4.3.2. Numerical Simulation II. The object is selected as Case 3. Figure 11(a) plots the time series of heave displacement by ANSYS/Fluent adopted in the present method and OpenFOAM. Similarly, two curve trends preliminarily validate the applicability of the present study. The average error in Figure 11(b) is 0.52, which further demonstrates that the 


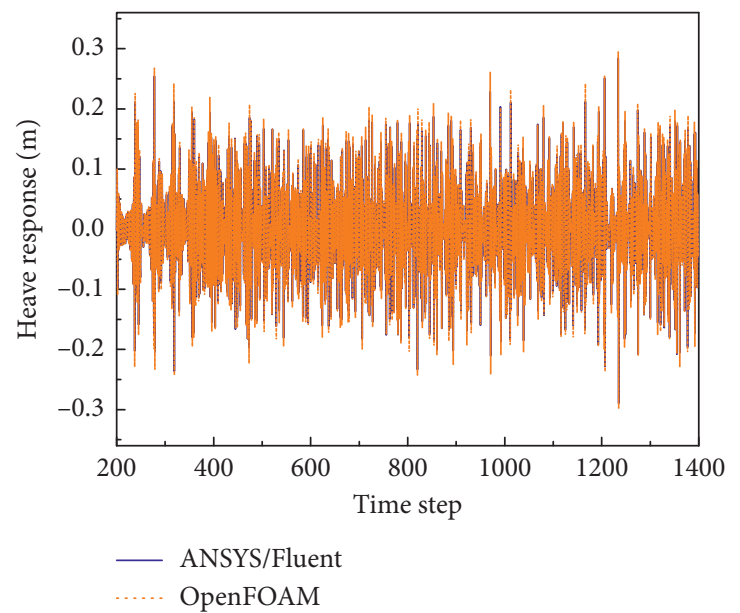

(a)



(b)

FIGURE 10: The heave response comparisons of the offshore wind turbine platform by ANSYS/Fluent and OpenFOAM: (a) the time series of heave displacement and (b) the average error and time cost ratio statistics compared to OpenFOAM.

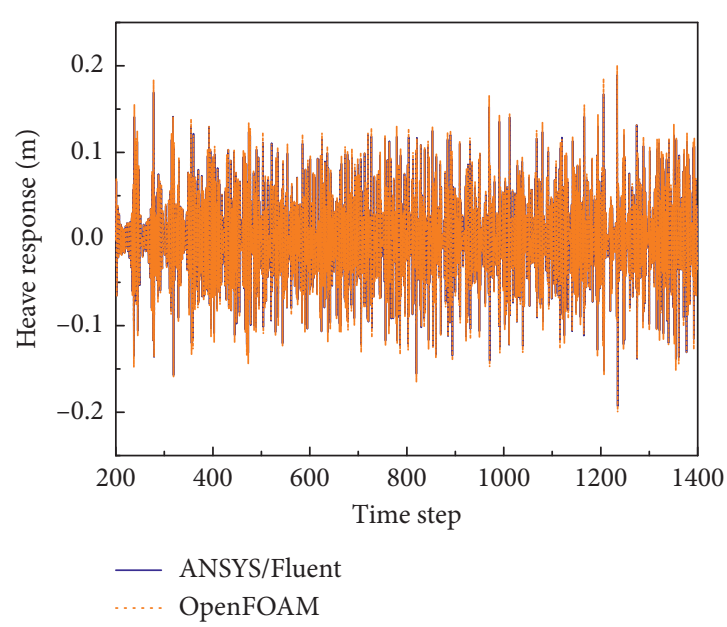

(a)



(b)

FIGURE 11: The heave response comparisons of Case 3 by the present and traditional methods: (a) the time series of heave displacement and (b) the average error and time cost ratio statistics compared to OpenFOAM.

tightly coupled VOF/6-DOF method compiled in Fluent UDF Library is accurate in the performance assessment of Case 3 with larger size.

In addition, Table 9 summarizes the $Z_{R M S}$ results from numerical simulations I and II. It can be concluded that the results of the two methods are basically the same. Therefore, the tightly coupled VOF/6-DOF method compiled in Fluent UDF Library is proved to be more accurate and stable than the traditional method to assess the towing and motion performances of Cases 1 to 6 .

\section{Numerical Examples}

In this section, numerical simulations are performed to evaluate the effects of different structural forms on the towing and motion performances under various environmental conditions.

5.1. Towing Performance Evaluation. The environmental conditions are listed as follows: the sea states, which refer to average wind speed, significant wave height, and wave period, are $11 \mathrm{~m} / \mathrm{s}, 3 \mathrm{~m}$, and $6 \mathrm{~s}$, while the towing speed $v_{t}$ is ranging from $1 \mathrm{kN}$ to $8 \mathrm{kN}$, with the interval $\Delta v_{t}=1 \mathrm{kN}$.

5.1.1. Pontoon End Shape. Under the combined loadings of wind and waves, Figure 12(a) depicts the relationship between the towing resistances of the platform and the towing speeds for three different pontoon end shapes. It is found that all the towing resistances of Cases 1 to 3 increase with the towing speeds. With a same platform draft, the rank of 
TABLE 9: RMS of heave response by ANSYS/Fluent and OpenFOAM.

\begin{tabular}{|c|c|c|c|c|}
\hline Case & \multicolumn{2}{|c|}{ Numerical simulation I } & \multicolumn{2}{|c|}{ Numerical simulation II } \\
\hline Method & ANSYS/Fluent & OpenFOAM & ANSYS/Fluent & OpenFOAM \\
\hline$Z_{R M S}$ & 0.0924 & 0.0932 & 0.0685 & 0.0691 \\
\hline
\end{tabular}



(a)



(b)



(c)

FIGURE 12: (a) The towing resistance of the platform at different towing speed, (b) the towing resistance coefficient of the pontoons in Cases 1 to 3 at the towing speed of $4 \mathrm{kN}$, and (c) the towing resistance of Case 3 at the towing speed of $4 \mathrm{kN}$.

the towing resistance is Case $3>$ Case $1>$ Case 2 . Compared to Case 3 (rounded cube), the towing resistance of Case 2 $\left(30^{\circ}\right.$ sharp angle) has an approximately $14.6 \%$ decrease. Moreover, Figure 12(b) especially records the time series of towing resistance coefficients of the pontoons only in Cases 1 to 3 at a towing speed of $4 \mathrm{kN}$. The time-varying resistance coefficients of the pontoons in Case 2 are always less than those of Cases 1 and 3. It similarly illustrates that Case 2 has the better towing performance.

However, as shown in Table 3, the pontoon length of Case 2 is $16.16 \mathrm{~m}$ longer than Case 3 and $13.38 \mathrm{~m}$ longer than Case 1. Thus, in case of a uniform design length of pontoon for all of the three cases, the draft of Case 2 will be much less than Cases 1 and 3. Under this assumption, Case 1 turns to be a relatively ideal scheme. Therefore, the pontoon end shape selection depends on both the towing performance and design requirements.

Figure 12(c) investigates the towing resistances of each structural component as well as the platform at the towing speed of $4 \mathrm{kN}$. The total towing resistance of Case 3 is $1310 \mathrm{kN}$. The rank of three structural components in ascending order is pontoons, columns, and horizontal connections (braces), which approximately occupy $45 \%, 33 \%$, and $22 \%$ of the total resistance, respectively. Therefore, in consideration of the towing performance optimization, the pontoon end shape should have priority over the other two components. 


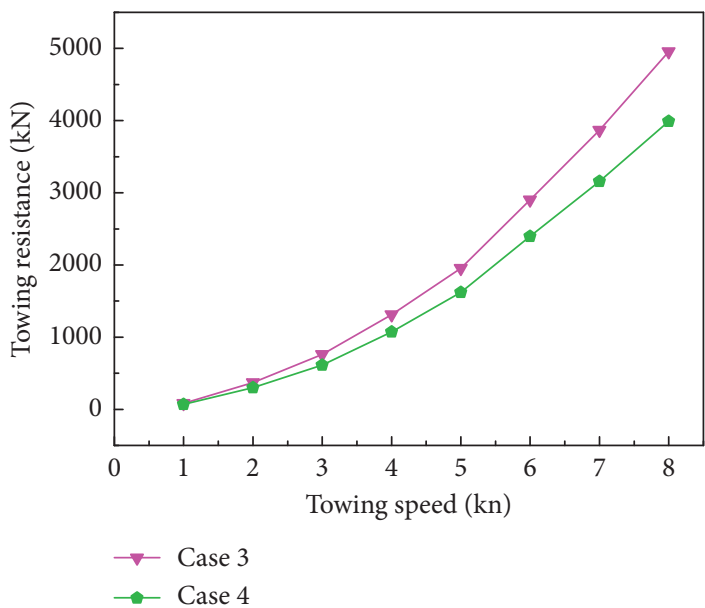

(a)



(b)

Figure 13: (a) The towing resistance of the platform at different towing speeds. (b) The towing resistance coefficient of different column cross sections at the towing speed of $4 \mathrm{kN}$.

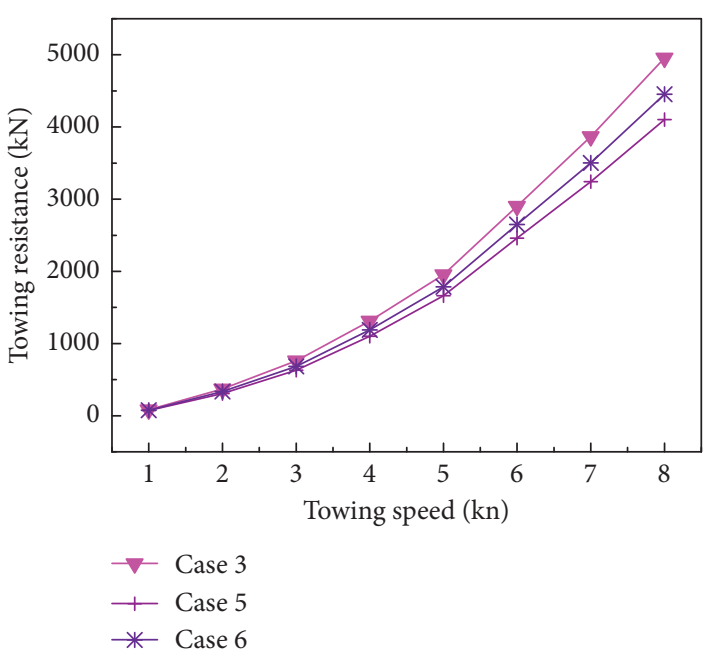

(a)



(b)

FIGURE 14: (a) The towing resistance of the platform at different towing speeds. (b) The towing resistance coefficient of the braces in Cases 3 , 5 , and 6 at the towing speed of $4 \mathrm{kN}$.

5.1.2. Column Cross Section. Figure 13(a) investigates the varying towing resistances of Cases 3 and 4 with different towing speeds. Both curves reveal that the towing resistance increases with the towing speed. Meanwhile, the resistances of Case 4 are always less than those of Case 3 with the differences ranging from $16.7 \%$ to $19.4 \%$. For the towing speed less than $4 \mathrm{kN}$, the towing resistances of Cases 3 and 4 have a little difference, while for speeds larger than $4 \mathrm{kN}$, the difference increases significantly.

Figure 13(b) records in detail the time series of the towing resistance coefficient of the columns only in Cases 3 and 4 at the towing speed of $4 \mathrm{kN}$. The towing resistance coefficients of the columns in Case 4 are all the time less than those in Case 3. Their maximum percentage of difference could reach $51.7 \%$. In view that the contribution of the columns to the total resistance is $33 \%$, the total maximum differences of the towing resistance coefficient between Cases 3 and 4 are about $17.1 \%$. The estimated result is relatively close to the result in Figure 13(a).

To sum up, compared to Case 3, Case 4 with a circle cross section has a better towing performance. The column selection still needs to take the draft and motion response into consideration.

5.1.3. Brace Longitudinal Section. Similarly, Figure 14(a) explores the regulation between the towing resistance and the towing speed among three different types of the brace 


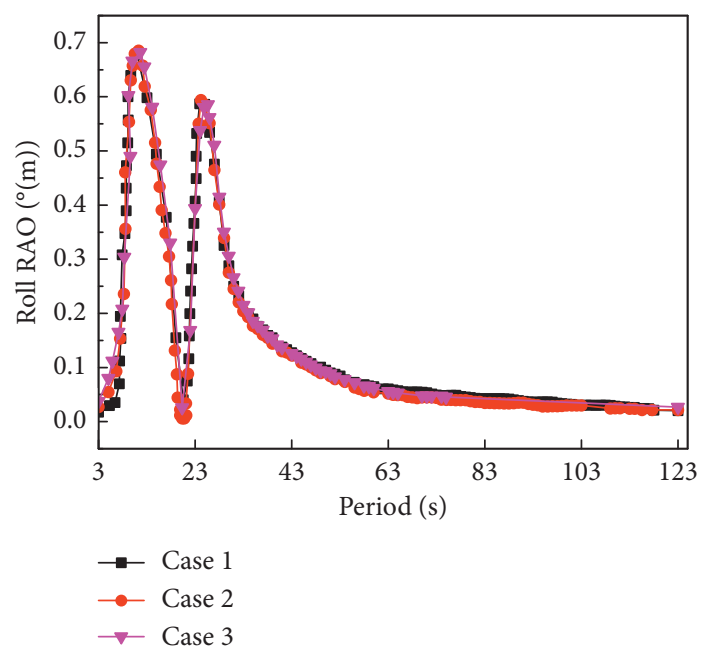

(a)

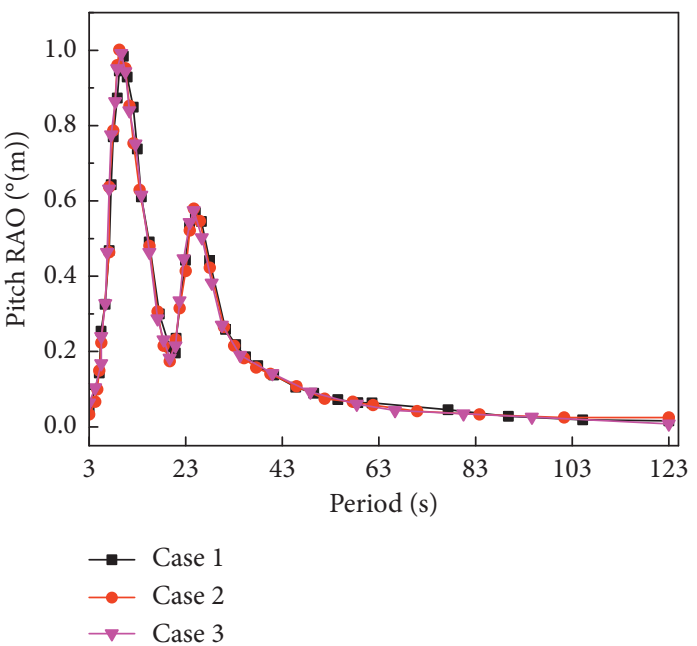

(b)



(c)

Figure 15: The motion response of the platform with different pontoon end shapes. (a) Roll, (b) pitch, and (c) heave.

longitudinal section. All the towing resistances of Cases 3, 5, and 6 increase with the towing speeds. Meanwhile, the towing resistances of Case 3 are larger than those of Cases 5 and 6. Compared to Case 3, the differences are $16.1 \%$ and $9.3 \%$, respectively. Figure 14 (b) records the time series of the towing resistance coefficient of braces only in Cases 3, 5, and 6 at the towing speed of $4 \mathrm{kN}$. The resistance coefficients of Cases 5 and 6 reduce approximately by $45.7 \%$ and $39.1 \%$, respectively, when compared to Case 3 . In view that the contribution of the braces to the total resistance is $22 \%$, the resistance of Cases 5 and 6 will have a $10 \%$ and $8 \%$ decrease than that of Case 3.

Therefore, the towing performance of the plate shape in Cases 5 and 6 is better than that of circle longitudinal section in Case 3.

5.2. Motion Performance Evaluation. The environmental conditions are listed as follows: the wind and wave direction is $0^{\circ}$, the wave period $T_{\text {wave }}$ ranges from $3 \mathrm{~s}$ to $123 \mathrm{~s}$, and interval of wave period $\Delta T_{\text {wave }}=1 \mathrm{~s}$ and $v_{t}=0$.
5.2.1. Pontoon End Shape. The periodic motion responses of Cases 1 to 3 are calculated by using time-frequency conversion method, as shown in Figure 15. All the curves for three cases in roll, pitch, and heave responses have the similar trend, and the response amplitudes of Cases 1 to 3 have no significant difference. The maximum roll response amplitudes of Cases 1 to 3 are 0.67788, 0.6853, and 0.6891 . The maximum pitch response amplitudes of Cases 1 to 3 are $0.9846,1.0014$, and 1.0012 and the maximum heave response amplitudes of Cases 1 to 3 are 1.9388, 1.9728, and 2.0137. Thus, it can be considered that the pontoon end shape has a little influence on the motion performance of the whole platform. In the pontoon design, the effect of pontoon end shape on the motion response can be neglected.

5.2.2. Column Cross Section. Distinct from the influence of pontoon end shape on motion performance, all the roll, pitch, and heave responses have the significant changes for different column cross sections, as shown in Figure 16. Seen from the roll and pitch periodic response curves in 


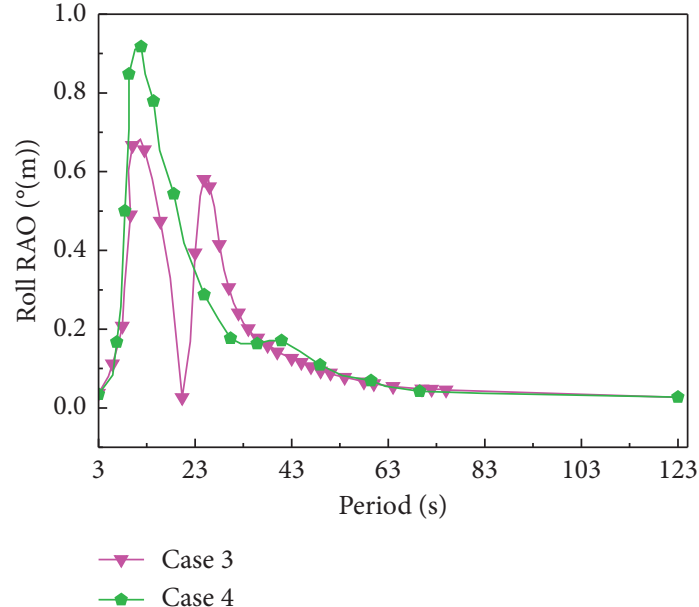

(a)

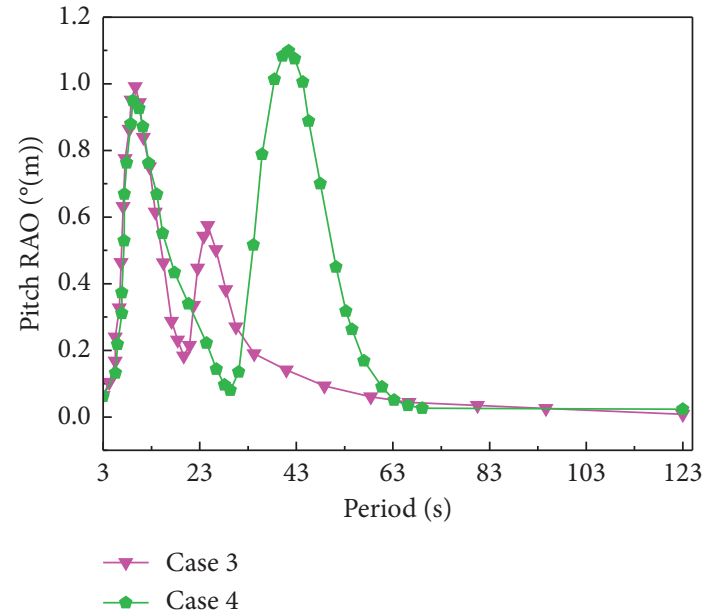

(b)

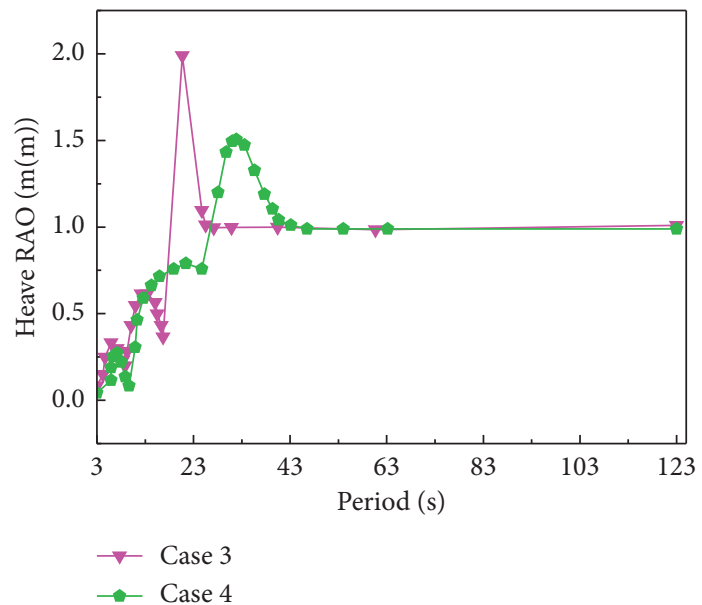

(c)

Figure 16: The motion response of the platform with different column cross sections. (a) Roll, (b) pitch, and (c) heave.



(a)



(b)

Figure 17: Continued. 


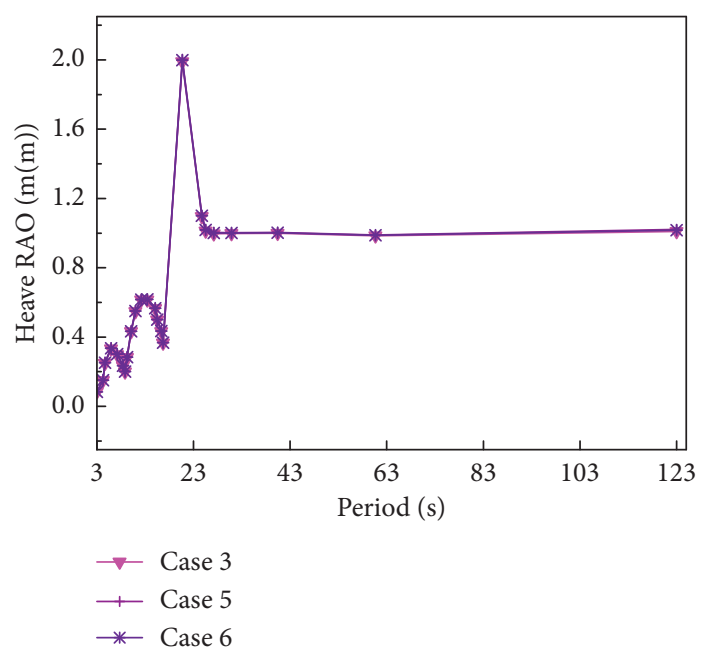

(c)

Figure 17: The motion response of the platform with different brace longitudinal sections. (a) Roll, (b) pitch, and (c) heave.

Figures 16(a) and 16(b), the response amplitudes of Case 4 with the circular cross section are obviously larger than those of Case 3 with the square cross section. Peak values for roll response are 0.91764 and 0.6823 for Case 4 and Case 3, while for the pitch response, they are 1.09901 and 0.99156 , which may break the safety criteria of the platform. In Figure 16(c), the heave response amplitudes of Case 4 are far smaller than those of Case 3, with values of 1.50526 and 1.9892 , which may reduce the possibility of waves against the deck structures and improve the safety of the platform.

In sum, for motion response, Case 3 and Case 4 have their own advantages and disadvantages. Thus, the column selection should be under a comprehensive consideration of the towing performance, the motion response requirements of each degree of freedom, et al.

5.2.3. Brace Longitudinal Section. Figure 17 plots the roll, pitch, and heave periodic responses of Cases 3, 5, and 6, whose brace longitudinal section differs from each other. Similar to the regulations of the pontoon end shape, all the curves are relatively close. The maximum roll response amplitudes of Cases 3, 5, and 6 are $0.6823,0.6835$, and 0.6831 . The maximum pitch response amplitudes of Cases 3 , 5 , and 6 are $0.9916,0.9969$, and 0.9949. And the maximum heave response amplitudes of Cases 3, 5, and 6 are 1.9892, 2.0032, and 1.9986. It is found that the peak response amplitudes have little difference and share the same corresponding wave periods. Therefore, Cases 3, 5, and 6 have the essentially similar motion performance.

In summary, the effect of the brace longitudinal section on the motion performance can be ignored. The brace design should only consider the towing performance and other design requirements.

\section{Conclusions}

In this study, the effects of different structural components on the towing and motion performances are investigated by using the integrated CFD method that contains RANS with SST k- $\omega$ turbulent model, a VOF/6-DOF solver (i.e., a joint application of VOF model and a tightly coupled 6-DOF solver), and a UDF Library for reading the time series of wave speed. The numerical simulations lead to the following conclusions:

(a) The VOF/6-DOF solver compiled in a new Fluent UDF Library has been validated to be accurate and stable. By comparing the heave responses of a wind turbine platform used in previous study and the production platform of Case 3 with different methods, it could be verified that the innovative solver adopted in the present study is relatively in agreement with OpenFOAM. It improves the computational stability and accuracy, compared with the traditional 6-DOF solver in ANSYS/Fluent

(b) Towing performances of Cases 1 to 6 are evaluated at the towing speeds ranging from $1 \mathrm{kN}$ to $8 \mathrm{kN}$. All the towing resistances increase with the towing speeds. The towing resistances of the platform components in ascending order are pontoons, columns, and braces, which share approximately $45 \%, 33 \%$, and $22 \%$ of the total resistance, respectively. Only from the perspective of towing performance, the following suggestions from the abovementioned in-depth investigation are listed as follows. For the pontoon end shape, the proper form is Case $2\left(30^{\circ}\right.$ sharp angle); however, in the case of a same platform draft, the proper form is Case 1 (half round). For the column cross section, Case 4 (circle) has a better motion 
performance than Case 3 (square) when the towing speed is greater than $4 \mathrm{kN}$. For the brace longitudinal section, the towing resistances of Cases 5 and 6 (plate shape) are relatively close and less than those of Case 3 (circle). Thus, the platform design should be optimally chosen as the plate shape

(c) Motion responses of Cases 1 to 6 are analyzed at 121 sea states (i.e., wave period ranges from $3 \mathrm{~s}$ to $123 \mathrm{~s}$ with an interval of $1 \mathrm{~s}$ ). Roll, pitch, and heave periodical responses lead to the following suggestions. Firstly, the pontoon end shapes and brace longitudinal sections have no significant influence on the motion response of the whole platform. Meanwhile, the motion responses of different column cross sections have great differences. Herein, the roll and pitch responses of Case 4 are greater than those of Case 3, which may threaten the safety of the platform. On the contrary, the heave responses of Case 4 are less than those of Case 3, which will improve the safety of the platform. Therefore, the column design should comprehensively consider the response amplitude limitations of each degree of freedom, the towing performance, and other design requirements.

\section{Data Availability}

The data used to support the findings of this study are included within the article. Previously reported data were used to support this study and are available at [10.1155/2017/ 3210271 and 10.21595/jve.2017.18872]. These prior studies (and datasets) are cited at relevant places within the text as references (J. Ma, D. Zhou, Z.L. Han, K. Zhang, J. Nguyen, J.B. Lu, and Y. Bao (2017); Numerical Simulation of Fluctuating Wind Effects on an Offshore Deck Structure. Shock and Vibration, 1:1-17; J. Ma, D. Zhou, Y. Bao, and Z.L. Han (2018); Fatigue assessment on local components of a semisubmersible platform subjected to wind and wave loads. Journal of Vibroengineering, 20(2): 988-1006).

\section{Disclosure}

The funders had no role in the design of the study; in the collection, analyses, or interpretation of data; in the writing of the manuscript; or in the decision to publish the results.

\section{Conflicts of Interest}

The authors declare no conflicts of interest regarding the publication of this paper.

\section{Acknowledgments}

This study was financially supported by the National Natural Science Foundation of China (Grants nos. 51879160 and 11772193), the Innovation Program of Shanghai Municipal Education Commission (Grant no. 2019-01-07-00-02E00066), the Shanghai Natural Science Foundation (Grant no. 18ZR1418000), and China Postdoctoral Science
Foundation (Grant no. 2020M671128). This research was also supported in part by the Program for Professor of Special Appointment (Eastern Scholar) at Shanghai Institutions of Higher Learning (Grant no. ZXDF010037), the Project of Thousand Young Talents (Grant no. BE0100002), the National Natural Science Funds for Distinguished Young Scholars (Grant no. 51825903), and the Joint Funds of the National Natural Science Foundation of China (Grant no. U19B2013).

\section{References}

[1] H. Zhu and J. Ou, "Dynamic performance of a semi-submersible platform subject to wind and waves," Journal of Ocean University of China, vol. 10, no. 2, pp. 127-134, 2011.

[2] X. Wang, J.-F. Zhou, Z. Wang, and Y.-X. You, "A numerical and experimental study of internal solitary wave loads on semi-submersible platforms," Ocean Engineering, vol. 150, pp. 298-308, 2018.

[3] DNV, Cn30-5 environmental conditions and environmental loads, vol. 3, DNV, Oslo, Norway, 2000.

[4] O. M. Faltinsen, Sea Loads on Ships and Offshore Structures, Cambridge University Press, Cambridge, UK, 1993.

[5] M. I. Kvittem, E. E. Bachynski, and T. Moan, "Effects of hydrodynamic modelling in fully coupled simulations of a semi-submersible wind turbine," Energy Procedia, vol. 24, pp. 351-362, 2012.

[6] A. J. Coulling, A. J. Goupee, A. N. Robertson, J. M. Jonkman, and H. J. Dagher, "Validation of a FAST semi-submersible floating wind turbine numerical model with DeepCwind test data," Journal of Renewable \& Sustainable Energy, vol. 5, no. 2, pp. 557-569, 2013.

[7] X. Wang, J. Zhou, Y. You, and Y. X. You, “A numerical wavemaker for internal solitary waves with timely updated mass source/sink terms," European Journal of Mechanics - B/Fluids, vol. 65, pp. 274-283, 2017.

[8] ANSYS Inc, AQWA Reference Manual, ANSYS Inc., Canonsburg, PA, USA, 2012.

[9] ANSYS Inc. Guide, ANSYS FLUENT Tutorial Guide, ANSYS Inc., Canonsburg, PA, USA, 2012.

[10] C. H. Lee and J. N. Newman, WAMIT User Manual, Vol. 6, WAMIT Inc., Chestnut Hill, MA, USA, 2006.

[11] J. Jonkman, Dynamics Modeling and Loads Analysis of an Offshore Floating Wind Turbine, ProQuest, Ann Arbor, MI, USA, 2007.

[12] T. T. Tran and D.-H. Kim, "The coupled dynamic response computation for a semi-submersible platform of floating offshore wind turbine," Journal of Wind Engineering and Industrial Aerodynamics, vol. 147, pp. 104-119, 2015.

[13] J. Ma, D. Zhou, Z. Han et al., "Numerical simulation of fluctuating wind effects on an offshore deck structure," Shock and Vibration, vol. 2017, Article ID 3210271, 17 pages, 2017.

[14] J. Ma, D. Zhou, Y. Bao, and Z. L. Han, "Fatigue assessment on local components of a semi-submersible platform subjected to wind and wave loads," Journal of Vibroengineering, vol. 20, no. 2, pp. 988-1006, 2018.

[15] A. J. Dunbar, B. A. Craven, and E. G. Paterson, "Development and validation of a tightly coupled CFD/6-DOF solver for simulating floating offshore wind turbine platforms," Ocean Engineering, vol. 110, pp. 98-105, 2015.

[16] A. R. Henderson and M. H. Patel, "On the modelling of a floating offshore wind turbine," Wind Energy, vol. 6, no. 1, pp. 53-86, 2003. 
[17] M. C. Bettle, A. G. Gerber, and G. D. Watt, "Unsteady analysis of the six DOF motion of a buoyantly rising submarine," Computers \& Fluids, vol. 38, no. 9, pp. 1833-1849, 2009.

[18] T. Schwarz, "RANS simulations with one and six degrees of freedom rigid body motions," in New Results in Numerical and Experimental Fluid Mechanics VI, C. Tropea, Ed., pp. 178-185, Springer-Verlag, Berlin, Heidelberg, 2007.

[19] Z. Yu, Y. Shen, J. Amdahl, and M. Greco, "Implementation of linear potential-flow theory in the 6DOF coupled simulation of ship collision and grounding accidents," Journal of Ship Research, vol. 60, no. 3, pp. 119-144, 2016.

[20] R. V. Wilson, P. M. Carrica, and F. Stern, "Unsteady rans method for ship motions with application to roll for a surface combatant," Computers \& Fluids, vol. 35, no. 5, pp. 501-524, 2006.

[21] J. Kim, A. Magee, and K. Guan, "CFD simulation of flowinduced motions of a multicolumn floating platform," in Proceeding of the ASME 2011 30th International Conference on Ocean, Offshore and Arctic Engineering, American Society of Mechanical Engineers, Rotterdam, The Netherlands, pp. 319-326, 2011.

[22] Q. Xu, J. Kim, T. Bhaumik, J. OSullivan, and J. Ermon, "Validation of HVS semisubmersible VIM performance by model test and CFD," in Proceedings of the ASME 2012 31st International Conference on Ocean, Offshore and Arctic Engineering, pp. 175-185, American Society of Mechanical Engineers, Rio de Janeiro, Brazil, July 2012.

[23] J. Tan, A. Magee, J. Kim, Y. Teng, and N. Zukni, "CFD simulation for vortex induced motions of a multi-column floating platform," in Proceeding of the ASME 2013 32nd International Conference on Ocean, Offshore and Arctic Engineering, American Society of Mechanical Engineers, Nantes, France, pp. 1-10, June 2013.

[24] P. Causin, J. F. Gerbeau, and F. Nobile, "Added-mass effect in the design of partitioned algorithms for fluid-structure problems," Computer Methods in Applied Mechanics and Engineering, vol. 194, no. 42-44, pp. 4506-4527, 2005.

[25] C. Förster, W. A. Wall, and E. Ramm, "Artificial added mass instabilities in sequential staggered coupling of nonlinear structures and incompressible viscous flows," Computer Methods in Applied Mechanics and Engineering, vol. 196, no. 7, pp. 1278-1293, 2007.

[26] R. Campbell, Fluid-structure Interaction and Inverse Design Simulations for Flexible Turbomachinery, Ph.D. thesis, The Pennsylvania State University, PA, USA, 2010.

[27] D. J. Piro and K. J. Maki, "Hydroelastic analysis of bodies that enter and exit water," Journal of Fluids and Structures, vol. 37, no. 37, pp. 134-150, 2013.

[28] C. Peyrard, P. Bousseau, R. Howard et al., "CFD modeling of flow induced vibration on a mobile cylinder for a $30 \mathrm{k}-60 \mathrm{k}$ Reynolds number comparison between simulation and experimental results," in Proceedings of the ASME 2009 Pressure Vessels and Piping Division Conference, pp. 301-308, Prague, Czech Republic, June 2009.

[29] B. L. Geng, Time domain simulation of wave action with sea platform in deep water, Ph.D. thesis, Dalian Univesity of Technology, Dalian, China, 2010. 